\title{
Endoscopic Management of Recurrent Anterior Skull Base Schwannoma
}

\author{
Jivianne T. Lee ${ }^{1,2}$, Lester D. R. Thompson ${ }^{3}$, Rohit Garg ${ }^{1}$, David B. Keschner ${ }^{1}$, Terry Shibuya ${ }^{1}$ \\ ${ }^{1}$ Orange County Sinus and Skull Base Institute, Southern California Permanente Medical Group, Irvine, USA \\ ${ }^{2}$ Department of Head \& Neck Surgery, David Geffen School of Medicine, University of California, Los Angeles, USA \\ ${ }^{3}$ Department of Pathology, Woodland Hills Medical Center, Southern California Permanente Medical Group, Woodland Hills, USA \\ Email: jivianne@gmail.com
}

Received February 6, 2013; revised March 11, 2013; accepted March 19, 2013

\begin{abstract}
Objectives: Sinonasal schwannomas account for less than $4 \%$ of head and neck schwannomas, with the primary treatment modality being surgical excision via external approaches. The aim of this report is to present a rare case of recurrent schwannoma of the ethmoid cavity involving the anterior skull base which was successfully managed with endoscopic resection. Study Design: Case report and review of the literature. Methods: The clinical presentation, radiographic features, histopathologic characteristics, surgical approach, and patient outcome were examined in the context of a literature review. Results: A 43-year-old woman presented with a 9-month history of left facial pain and pressure. She had a prior history of sinonasal schwannoma excision with cerebrospinal fluid (CSF) leak repair via bifrontal craniotomy in 2007. Magnetic resonance imaging (MRI) and nasal endoscopy revealed a left ethmoid mass measuring 2.2 $\mathrm{cm} \times 2.7 \mathrm{~cm} \times 2.4 \mathrm{~cm}$ abutting the anterior skull base. The tumor was completely removed using a transnasal endoscopic approach, and the anterior skull base reconstructed with tensor fascia lata graft. Histology of the specimen showed schwannoma, and there has been no evidence of tumor recurrence nor CSF leak after 24 months of follow-up. Conclusion: With continual advances in surgical technique and instrumentation, sinonasal schwannomas have become increasingly more amenable to endoscopic resection even in the case of recurrence and skull base involvement.
\end{abstract}

Keywords: Endoscopic; Recurrent; Anterior Skull Base; Sinonasal; Schwannoma

\section{Introduction}

First described by Virchow in 1908, schwannomas (peripheral nerve sheath tumors) are benign, slow-growing, well-encapsulated tumors that arise from Schwann cells, derived from ectoderm (neural crest), which provide myelin insulation for peripheral motor, sensory, autonomic, and cranial nerves [1]. Although $25 \%$ - $45 \%$ occur in the head and neck, sinonasal tract involvement is unusual, accounting for $<4 \%$ of all head and neck schwannomas [2]. The nasoethmoid complex is the most commonly affected site, followed by the maxillary sinus, nasal cavity, sphenoid sinus, and frontal sinus [2,3].

Traditionally, the primary treatment modality has been surgical excision via external procedures [4]. However, recent advances in endoscopic techniques have resulted in an increasing number of cases being managed with less invasive methods [4]. We present a unique case of recurrent schwannoma of the ethmoid cavity and anterior skull base successfully treated with endoscopic resection and reconstruction of the skull base defect. The clinical presentation, radiographic findings, pathologic features, surgical approach, treatment, and outcome are discussed.

\section{Case Report}

A 43-year-old woman was referred to our clinic in December 2010 complaining of left facial pain and pressure for 9 months. She also noted a 3-week history of clear postnasal drainage which she described as being predominantly left-sided and "salty" in flavor. The patient denied having any nasal obstruction, nasal congestion, headache, or epistaxis. Her prior surgical history was significant for excision of a left nasoethmoid schwannoma and cerebrospinal fluid leak (CSF) repair via bifrontal craniotomy in 2007 at a different facility. Postoperatively, the patient experienced longstanding anosmia but had no additional symptoms until the time of presentation.

On nasal endoscopy the patient was found to have a left-sided, smooth, yellow/pink-colored mass abutting the nasal septum which extended superiorly towards the ethmoid roof (Figure 1). The middle turbinate appeared to have been previously resected. No clear discharge was visualized in the left nasal cavity nor nasopharynx. The patient was also unable to produce a specimen for beta-2 transferrin analysis to confirm the presence of CSF rhi- 
norrhea. However, the patient reported experiencing similar symptoms at the time of her initial diagnosis and subsequent CSF leak. Radiographic studies were ordered, with a computed tomography (CT) scan showing a left ethmoid sinus mass approximating the anterior skull base (Figure 2). No bony erosion nor skull base dehiscence was appreciated. The remaining paranasal sinuses were otherwise unremarkable. Magnetic resonance imaging (MRI) revealed a $2.2 \mathrm{~cm} \times 2.7 \mathrm{~cm} \times 2.4 \mathrm{~cm}$ homogeneously enhancing, smoothly-marginated soft-tissue lesion within the left anterior ethmoid cavity which extended from the skull base to the level of the superior aspect of the inferior turbinate (Figure 3). No intracranial involvement was detected.

The patient elected to proceed with excision of the mass with possible CSF leak repair using an endoscopic transnasal approach. Triplanar stereotactic imaging was obtained in preparation for computer-assisted surgical navigation. Intraoperatively, a biopsy was sent for frozen section, interpreted as schwannoma, clinically recurrent. A complete ethmoidectomy, sphenoidotomy, and frontal sinusotomy were then performed to expose the boundaries of the lesion. Upon inspection with 30- and 70-degree $4 \mathrm{~mm}$ telescopes, the tumor was found to be attached to the superior aspect of the septum and cribriform plate.

Endoscopic instruments were employed to resect the lesion en-bloc, including a rim of normal appearing respiratory mucosa. Multiple small defects with areas of CSF drainage was noted within the ethmoid roof at the site of pedicle attachment. The anterior skull base was then reconstructed using tensor fascia lata graft harvested from the left thigh. The final pathology demonstrated characteristic features of schwannoma, including cellular Antoni A and hypocellular Antoni B areas (Figure 4).

Vessel wall sclerosis/hyalinization was noted. The cells

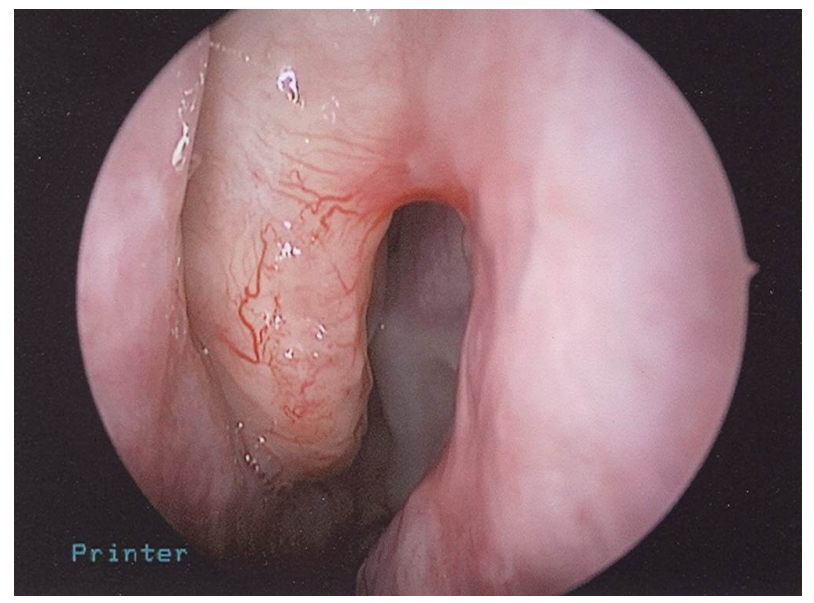

Figure 1. Preoperative nasal endoscopy revealed a left-sided, smooth, yellow/pink-colored mass abutting the nasal septum.

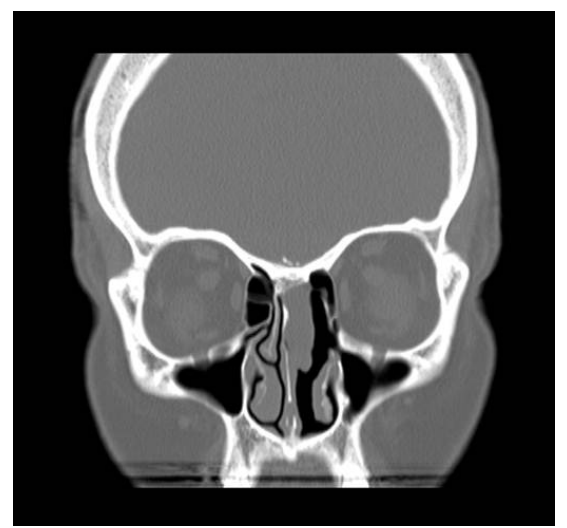

(a)

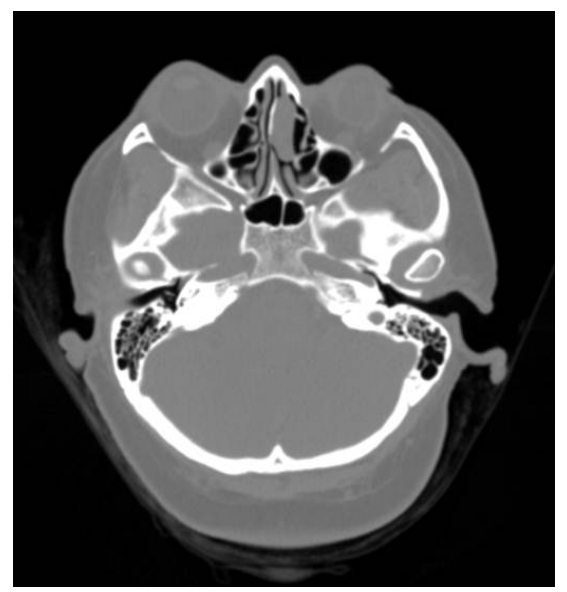

(b)

Figure 2. Preoperative (a) coronal and (b) axial computed tomography images demonstrated a mass within the left ethmoid cavity approximating the anterior skull base.

were spindled with elongated cytoplasmic extensions, and wavy nuclei. There was no pleomorphism, increased mitoses, nor necrosis. The neoplastic cells demonstrated strong, diffuse, nuclear and cytoplasmic reactivity with S100 protein immunohistochemistry (Figure 5). At last follow-up (24 months), there was no evidence of tumor recurrence nor CSF leak.

\section{Discussion}

Schwannomas are benign, well-encapsulated, solitary neoplasms which develop from Schwann cells that comprise the neural sheath of peripheral myelinated nerve fibers and envelope cranial nerve axons as they exit the central nervous system [1]. The origin of sinonasal schwannomas is presumed to be the ophthalmic and maxillary branches of the trigeminal nerve as well as autonomic ganglia, including the parasympathetic fibers of the sphenopalatine ganglion and the sympathetic fibers of the carotid plexus [5]. The actual nerves of origin are often extremely difficult to identify both pre- and intraoperatively due to their small size [3-5]. Ethmoid sch- 


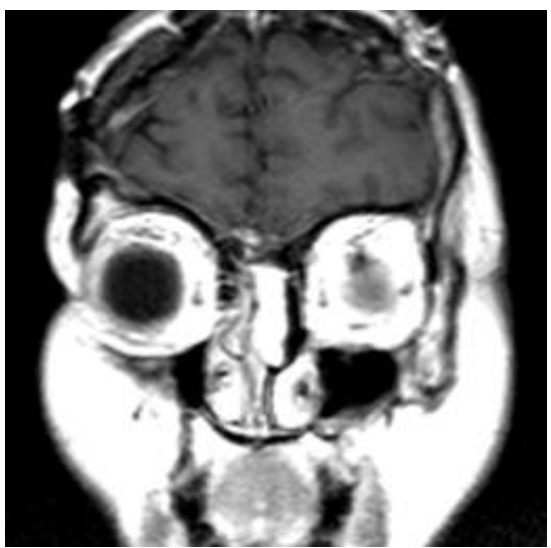

(a)

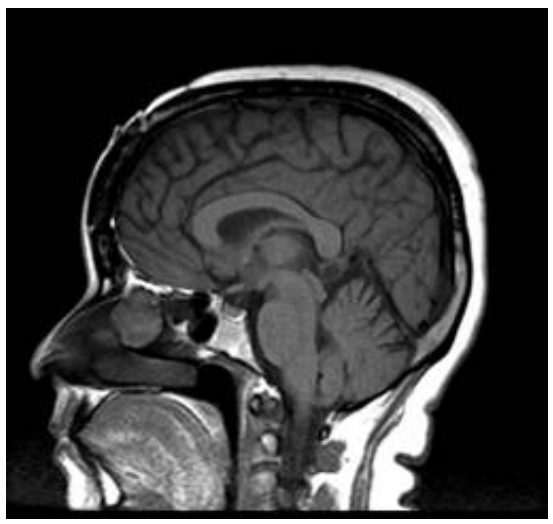

(b)

Figure 3. Preoperative (a) coronal T1-Magnetic Resonance Image (MRI) postcontrast and (b) sagittal T1-MRI showed a homogeneously enhancing, smoothly-marginated softtissue lesion $(2.2 \mathrm{~cm} \times 2.7 \mathrm{~cm} \times 2.4 \mathrm{~cm})$ within the left anterior ethmoid cavity extending from the skull base to the level of the superior aspect of the inferior turbinate.

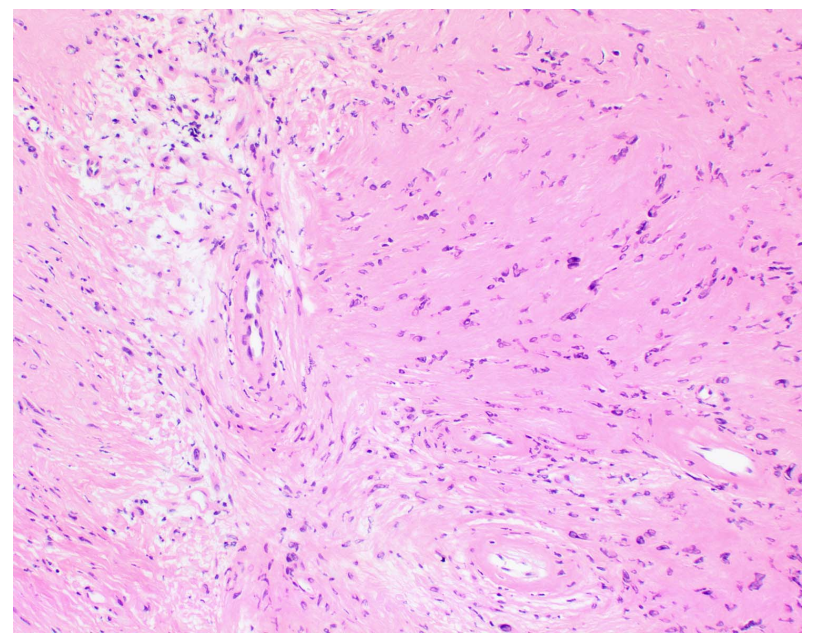

Figure 4. Photomicrograph $(100 \times)$. The left side shows an Antoni B (hypocellular) area which is adjacent to a slightly more cellular, Antonio A area. Note the three vessels in the illustration have a perivascular hyalinization characteristic of schwannoma.

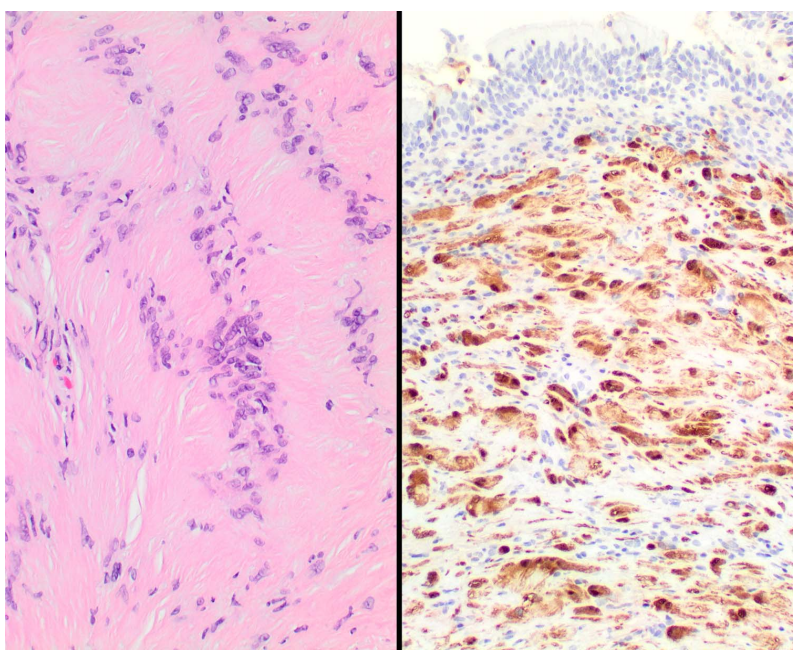

Figure 5. Photomicrograph $(400 \times)$. Left: A palisade of tumor nuclei create a verrocay body. Right: There is a strong and diffuse nuclear and cytoplasmic $\mathrm{S} 100$ protein reaction in the schwann cells. Note the respiratory epithelium at the top of the field (uninvolved).

wannomas involving the anterior skull base are believed to originate from the anterior ethmoid branch of the ophthalmic division of the trigeminal nerve, which was the likely scenario for this patient [5].

Clinically, patients present with varied, nonspecific symptoms similar to those seen with other sinonasal tract diseases. Consequently, diagnosis is often delayed [6]. Nasal obstruction, rhinorrhea, hyposmia, epistaxis, and facial pain are the most common presenting complaints [7]. Proptosis, epiphora, visual problems, and cranial nerve palsies may also be evident depending on the location and extent of the tumor $[7,8]$. Facial pain is typically more often seen in patients with schwannomas of the maxillary sinus and pterygopalatine fossa, whereas nasal obstruction and epistaxis are more commonly observed in lesions of the nasoethmoid complex [9]. Sphenoid sinus schwannomas have been associated with headache and diplopia, particularly with involvement of the 3rd, 4th, and 6th cranial nerves [8]. In our patient, ipsilateral facial pain was the predominant symptom despite the absence of maxillary sinus and pterygopalatine fossa extension. Although the tumor may occur at any age, there is a peak incidence between the 4th - 6th decades [10]. No gender or race predilection has been reported [8].

Upon nasal endoscopy, a unilateral polypoid mass is typically visualized. Imaging studies reveal nonspecific features, with schwannomas exhibiting a heterogeneous appearance on contrast-enhanced CT [11]. Mottled central lucency and peripheral intensification is often seen secondary to areas of neovascularity and adjacent necrosis [11]. Bony erosion is uncommon, but when present may be due to pressure necrosis from the gradually enlarging mass $[5,10]$. MRI is complementary to $\mathrm{CT}$ as it 
helps to distinguish the soft tissue neoplasm from inflammatory changes and retained secretions as well as aid in the evaluation of orbital and intracranial extension [11]. Sinonasal schwannomas usually demonstrate intermediate-high (isointense: Antoni A; hypointense: Antoni B) intensity on T1- and T2-weighted MRIs depending on the cystic characteristics of the lesion [4,7]. Gadolinium enhancement is also evident [11].

The differential diagnosis of paranasal sinus schwannomas include glial neoplasms, Schneiderian papillomas, olfactory neuroblastomas, neurofibromas, carcinomas, sarcomas, and lymphomas [7]. Schwannomas are welldefined, oval to fusiform lesions that expand along the course of peripheral nerves, pushing axons to the side as opposed to entrapping them [3]. Biopsy with pathologic analysis provides definitive diagnosis. Histologically, schwannomas exhibit two patterns: Antoni A (hypercellular, fasciculated, organized) and Antoni B (hypocellular, reticular, edematous). Antoni A areas contain densely compact, palisading, spindle-shaped cells with hyperchromatic nuclei and indistinct cytoplasmic borders arranged in interlacing fascicles. Parallel aligned nuclei (Verocay bodies) surrounded by fibrillary cell processes may also be present (Figure 5). The Antoni B blend or are adjacent to Antoni A regions, showing a more loose to myxoid stroma with fewer cells. There are also areas of perivascular hyalinization [1,5,10]. Schwannomas nearly always demonstrate strong and diffuse, nuclear and cytoplasmic S100 protein immunoreactivity, supporting the neuroectodermal derivation.

Although usually benign and well-circumscribed, sch- wannomas may spread to invade adjacent structures through direct extension and bony erosion [7]. In exceedingly rare instances, malignant transformation has also been reported [7]. Total surgical excision is considered to be the treatment of choice, with attempted preservation of critical anatomical structures. Schwannomas are often vascular and can be associated with significant bleeding during diagnostic biopsy and/or endoscopic excision, although estimated blood loss was about 25cc in the current case [4]. Recurrence is seldom seen following complete resection. With respect to sinonasal and anterior skull base lesions, a variety of external approaches have been advocated in the past including frontal craniotomy, lateral rhinotomy, craniofacial resection, Caldwell-Luc, midface degloving, external ethmoidectomy, and mixed combinations of these surgeries [4,7]. Such open procedures were driven by the need for adequate exposure as well as the location, size, and extent of the lesion. However, in recent years, the development of more advanced endoscopic surgical techniques has resulted in the successful removal of sinonasal schwannomas using less invasive methods [4,9]. The decreased morbidity, diminished blood loss, improved cosmesis, and shorter hospital stays associated with endoscopic sinonasal procedures have popularized their use over traditional, open approaches [4,9].

A review of published cases of ethmoid sinus schwannomas managed with an exclusively transnasal endoscopic approach are summarized in Table 1 Only two reports specifically described removal of lesions extending to the anterior skull base $[5,10]$. Hegazy, et al. presented

Table 1. Literature summary of ethmoid sinus schwannomas resected via an exclusively endoscopic transnasal approach.

\begin{tabular}{|c|c|c|c|c|c|c|}
\hline Author & Year & \# of cases & Age/Gender & Extent & Procedure & Follow-up (in years) \\
\hline $\begin{array}{c}\text { Blokmanis } \\
\text { [12] }\end{array}$ & 1994 & 1 & $46 \mathrm{M}$ & Right ethmoid/MT & Complete endoscopic removal & NED (4) \\
\hline Pasquini [9] & 2002 & 1 & $75 \mathrm{M}$ & Left anterior ethmoid/MT & $\begin{array}{l}\text { Lt partial middle turbinectomy, } \\
\text { total ethmoidectomy }\end{array}$ & NED (0.42) \\
\hline Pata [13] & 2005 & 1 & $66 \mathrm{M}$ & $\begin{array}{l}\text { Left ethmoid, maxillary, sphenoid } \\
\text { \& posterior NC }\end{array}$ & $\begin{array}{l}\text { Lt ESS, mass delivered thru } \\
\text { nasopharynx due to size }\end{array}$ & NED (0.75) \\
\hline Gillman [6] & 2005 & 1 & $52 \mathrm{~F}$ & Left MT \& posterior ethmoid & $\begin{array}{l}\text { Lt partial middle turbinectomy, } \\
\text { total ethmoidectomy }\end{array}$ & NED (3) \\
\hline Galli [3] & 2008 & 1 & $20 \mathrm{M}$ & $\begin{array}{l}\text { Right posterior ethmoid, NC, } \\
\text { choanae, erosion of septum, no } \\
\text { orbital or skull base involvement }\end{array}$ & Complete endoscopic removal & NED (0.75) \\
\hline Kodama [14] & 2010 & 1 & $81 \mathrm{~F}$ & Left NC (MT, superior meatus) & Lt ESS & NED (2) \\
\hline Suh [4] & 2011 & 3 & 51M, 68F, 49F & Ethmoid & Complete endoscopic removal & NED $(0.5-4.4)$ \\
\hline Adam [5] & 2012 & 1 & $51 \mathrm{M}$ & $\begin{array}{l}\text { Left middle meatus from } \\
\text { anterior skull base to nasal floor; } \\
\text { no skull base erosion }\end{array}$ & Lt MT resection, ESS & Not available \\
\hline
\end{tabular}

$\mathrm{M}=$ male, $\mathrm{F}$ = female, $\mathrm{MT}$ = middle turbinate, $\mathrm{NC}$ = nasal cavity, ESS = endoscopic sinus surgery, NED = no evidence of recurrent disease. 
a patient with schwannoma of the left ethmoid sinus which eroded the medial orbital wall and anterior cranial base [10]. Intraoperatively, leakage of CSF was observed along with multiple, small dural defects. The tumor was resected using endoscopic sinus surgical techniques and the dehiscences repaired using a middle turbinate mucosal flap. There was no tumor recurrence nor CSF leak evident 7 months after the surgery. Likewise, Adam and Vining reported a case of an anterior ethmoid schwannoma involving the skull base which filled the entire left nasal cavity from sphenoid ostium to frontal recess [5]. There was no evidence of skull base dehiscence, and the lesion was completed removed using an endoscopic, transnasal procedure under image guidance. Functional endoscopic sinus surgery was also performed to drain mucopurulence within the adjacent obstructed sinuses. No follow-up information was reported. Similarly, in our case, endoscopic surgical techniques were successfully implemented to excise a recurrent ethmoid schwannoma and reconstruct the anterior skull base.

\section{Conclusion}

Schwannomas are benign, slow-growing tumors that infrequently involve the nasal cavity and paranasal sinuses. Although uncommon, direct extension with bony erosion of the anterior skull base may occur. With the advent of more advanced endoscopic techniques and instrumentation, such lesions have become increasingly more amenable to transnasal endoscopic removal with minimal morbidity. Thus, endoscopic resection can be a safe and effective approach for surgical management of ethmoid sinonasal schwannomas involving the anterior skull base.

\section{REFERENCES}

[1] K. H. Perzin, H. Panyu and S. Wechter, "Nonepithelial Tumors of the Nasal Cavity, Paranasal Sinuses, and Nasopharynx: A Clinicopathologic Study, XII: Schwann Cell Tumors (Neurilemoma, Neurofibroma, Malignant Schwannoma)," Cancer, Vol. 50, No. 10, 1982, pp. 21932202.

doi:10.1002/1097-0142(19821115)50:10<2193::AID-CN CR2820501036>3.0.CO;2-0

[2] J. G. Batsakis, "Tumors of the Peripheral Nervous System," In: Tumors of the Head and Neck, 2nd Edition, Williams \& Wilkins, Baltimore, 1979, pp. 313-333.

[3] J. Galli, I. M. Imperiali, I. Cantore, L. Corina, L. M. La- rocca and G. Paludetti, "Atypical Sinonasal Schwannomas: A Difficult Diagnostic Challenge,” Auris Nasus Laynx, Vol. 36, No. 4, 2009, pp. 482-486.

doi:10.1016/j.anl.2008.11.010

[4] J. D. Suh, V. R. Ramakrishnan, P. J. Zhang, A. W. Wu, M. B. Wang, J. N. Palmer and A. G. Chiu, "Diagnosis and Endoscopic Management of Sinonasal Schwannomas,” ORL, Vol. 73, No. 6, 2011, pp. 308-312. doi:10.1159/000331923

[5] S. I. Adam and E. M. Vining, "Endoscopic Resection of an Anterior Skull-Base Schwannoma," International Forum of Allergy \& Rhinology, Vol. 2, No. 3, 2012, pp. 264 268. doi:10.1002/alr.21012

[6] G. Gillman and P. C. Bryson, "Ethmoid Schwannoma," Otolaryngology-Head and Neck Surgery, Vol. 132, No. 2, 2005, pp. 334-335. doi:10.1016/j.otohns.2004.04.027

[7] R. T. Younis, C. W. Gross and R. H. Lazar, "Schwannomas of the Paranasal Sinuses: Case Report and Clinicopathologic Analysis,” Archives of Otolaryngology-Head \& Neck Surgery, Vol. 117, No. 6, 1991, pp. 677-680. doi:10.1001/archotol.1991.01870180113022

[8] T. C. Calcaterra, R. Rich and P. W. Ward, "Neurilemmoma of the Sphenoid Sinus," Archives of Otolaryngology, Vol. 100, No. 5, 1974, pp. 383-385. doi:10.1001/archotol.1974.00780040395016

[9] E. Pasquini, V. Sciarretta, G. Farneti, A. Ippolito, D. Mazzatenta and G. Frank, "Endoscopic EndoNasal Approach for the Treatment of Benign Schwannoma of the Sinonasal Tract and Pterygopalatine Fossa," The American Journal of Surgery, Vol. 16, No. 2, 2002, pp. 113-8.

[10] H. M. Hegazy, C. H. Snyderman, C. Y. Fan and A. B. Kassam, "Neurilemmomas of the Paranasal Sinuses," American Journal of Otolaryngology-Head and Neck Medicine and Surgery, Vol. 22, No. 3, 2001, pp. 215-218. doi:10.1053/ajot.2001.23434

[11] E. Yu, D. Mikulis and S. Nag, "CT and MR imaging Findings in Sinonasal Schwannoma,” American Journal of Neuroradiology, Vol. 27, No. 4, 2006, pp. 929-930.

[12] A. Blokmanis, "Endoscopic Diagnosis, Treatment, and Follow-Up of Tumors of the Nose and Sinuses," Journal of Otorhinolaryngology, Vol. 2, No. 5, 1994, pp. 366-9.

[13] Y. S. Pata, Y. Akbas, M. Unal and C. Tataroglu, “A Case of Intranasal Schwannoma with Bilateral Nasal Polyposis," Kulak Burun Boğaz İhtisas Dergisi, Vol. 15, No. 1-2, 2005, pp. 45-48.

[14] S. Kodama, T. Okamoto and M. Suzuki, "Sinonasal Schwannoma with New Bone Formation Expressing Bone Morphogenic Protein,” International Journal of Otolaryngology, Vol. 2010, 2010, p. 154948. 